University of Nebraska - Lincoln

DigitalCommons@University of Nebraska - Lincoln

August 1987

\title{
Transition-Matrix Theory for Two-Photon Ionization of Rare-Gas Atoms and Isoelectronic lons with Application to Argon
}

Anthony F. Starace

University of Nebraska-Lincoln, astarace1@unl.edu

Tsin-Fu Jiang

University of Nebraska - Lincoln

Follow this and additional works at: https://digitalcommons.unl.edu/physicsstarace

Part of the Physics Commons

Starace, Anthony F. and Jiang, Tsin-Fu, "Transition-Matrix Theory for Two-Photon Ionization of Rare-Gas Atoms and Isoelectronic lons with Application to Argon" (1987). Anthony F. Starace Publications. 33. https://digitalcommons.unl.edu/physicsstarace/33

This Article is brought to you for free and open access by the Research Papers in Physics and Astronomy at DigitalCommons@University of Nebraska - Lincoln. It has been accepted for inclusion in Anthony F. Starace Publications by an authorized administrator of DigitalCommons@University of Nebraska - Lincoln. 


\title{
Transition-matrix theory for two-photon ionization of rare-gas atoms and isoelectronic ions with application to argon
}

\author{
Anthony F. Starace* \\ Institut für Theoretische Physik, Universität Innsbruck, A-6020 Innsbruck, Austria \\ and Department of Physics and Astronomy, The University of Nebraska, Lincoln, Nebraska 68588-0111 \\ Tsin-Fu Jiang ${ }^{\dagger}$ \\ Department of Physics and Astronomy, The University of Nebraska, Lincoln, Nebraska 68588-0111
}

(Received 18 February 1986; revised manuscript received 26 March 1987)

\begin{abstract}
A transition-matrix theory for two-photon ionization processes in rare-gas atoms or isoelectronic ions is presented. Uncoupled ordinary differential equations are obtained for the radial functions needed to calculate the two-photon transition amplitude. The implications of these equations are discussed in detail. In particular, the role of correlations involving virtually excited electron pairs, which are known to be essential to the description of single-photon processes, is examined for multiphoton ionization processes. Additionally, electron scattering interactions between two electron-hole pairs are introduced into our transition amplitude in the boson approximation since these have been found important in two-photon ionization of xenon by L'Huillier and Wendin [J. Phys. B 20, L37 (1987)]. Application of our theory is made to two-photon ionization of the $3 p$ subshell of argon below the one-photon ionization threshold. Our results are compared to previous calculations of McGuire [Phys. Rev. A 24, 835 (1981)], of Moccia, Rahman, and Rizzo [J. Phys. B 16, 2737 (1983)], and of Pindzola and Kelly [Phys. Rev. A 11, 1543 (1975)]. Results are presented for both circularly and linearly polarized photons. Among our findings are, firstly, that the electron scattering interactions, which have not been included in previous calculations for argon, produce a substantial reduction in the two-photon single-ionization cross section below the one-photon ionization threshold, which is in agreement with findings of L'Huillier and Wendin for xenon. Secondly, we find that deexcitation of virtually excited electron pairs by absorption of a photon is important for describing the interaction of the atom with the photon field, as in the case of single-photon ionization processes, but that further excitation of virtually excited electron pairs by the photon field has completely negligible effects, indicating a major simplification of the theory for higher-order absorption processes.
\end{abstract}

\section{INTRODUCTION}

Recent experiments on multiphoton ionization (MPI) ${ }^{1}$ of rare gases and alkaline earth atoms have revealed unexpectedly new behavior of multielectron atoms in strong laser fields. One striking example is the unusually large number of multiply charged ions produced by MPI of such atoms, each of which must have absorbed a large amount of energy, which in some cases is more than 200 $\mathrm{eV}^{2-5}$ Evidence of such large energy absorption in MPI has also been obtained by means of photoelectron spectroscopy; ${ }^{6,7}$ in xenon, for example, photoelectrons have been observed with kinetic energies corresponding to the absorption of 11 photons. $^{7}$ Theory is only beginning to understand this unexpectedly strong nonlinear response of multielectron atoms to strong radiation fields.

Experiments clearly indicate that electron correlation effects play a fundamental role in general in MPI and in particular in explaining these new results. As a result there is a renewed interest in theoretical descriptions of multiphoton absorption processes which go beyond the independent-particle model and include the treatment of electron correlations. One approach attempts to formulate a theory of MPI of complex atoms in which the interaction with the laser field is treated perturbatively. This approach may be considered as the natural extension of the theory of single-photon ionization ${ }^{8-10}$ to the multiphoton case, with its main emphasis the description of atomic correlation effects rather than laser intensity effects. The new problem in the calculation of nonresonant $n$-photon cross sections is that-in addition to describing the initial and final states of the atom $-n-1$ summations over the generally infinite set of intermediate states have to be performed. ${ }^{11}$ An accurate description of these virtual intermediate states requires treatment of electron correlation effects, in particular as these affect electric dipole matrix elements. The few theoretical calculations for multiphoton ionization which go beyond the HartreeFock approximation have treated the rare gases ${ }^{12-17}$ and the negative hydrogen ion. ${ }^{18,19}$ These calculations are described briefly below.

Other approaches focus on the description of MPI in the case of very intense laser fields for which a perturbative approach is not appropriate and for which therefore single-photon ionization is not so reliable a guide. Rhodes, ${ }^{4}$ for example, has postulated a model in which the outer atomic subshells are driven collectively by the laser field. At present, however, there is a growing consensus that currently observed multiply charged ion spectra may be understood as produced by sequential ionization in which each step may be treated theoretically within lowest-order perturbation theory (LOPT). ${ }^{20-25}$ 
This most recent work has therefore refocused attention on the role of electron correlations within LOPT.

In this paper we present a transition-matrix theory for nonresonant two-photon ionization of rare-gas atoms and isoelectronic ions within LOPT. From the theoretical point of view, the rare gases and rare-gas-like negative ions are the best candidates for developing a unified theoretical treatment of electron correlation effects on MPI processes. Firstly, the electron correlation problem for these atoms and ions is well understood in the case of single-photon ionization processes. ${ }^{8-10}$ This understanding is of great benefit in studying this new process. Secondly, these atoms' spherically symmetric ground states and lack of low-lying excited states simplify the theoretical treatment. Thirdly, the development of ArF excimer lasers has permitted the experimental measurement of the absolute two-photon ionization coefficient of xenon at $193 \mathrm{~nm}^{26}$

Our theory combines the differential equation method of Dalgarno and Lewis ${ }^{27}$ for summing over intermediate states with a generalization of the transition-matrix approaches of Chang and Fano ${ }^{28,29}$ and of Starace and Shahabi ${ }^{30-32}$ for treating electron correlations in singlephoton transitions. In single-photon ionization of the heavier rare gases, virtual excitations of pairs of outer $p$ subshell electrons in the initial state to excited $d$ orbitals are known to have significant effects on the calculated photoionization cross sections and angular distributions. ${ }^{8-10}$ It remains an open question in multiphoton ionization processes, however, whether such virtual excitations are also needed to describe the various intermediate states. On the answer to this question hangs the complexity of the theory required to describe accurately multiphoton ionization processes. Our transition-matrix theory presented here has been formulated to enable a straightforward answer to this question for two-photon ionization processes. Our calculations for two-photon ionization of argon, presented here, indicate such virtual excitations in intermediate states have negligible effects, thus simplifying considerably the development of theoretical extensions to three and more photon ionization processes.

In our transition-matrix theory we consider only the so-called particle-hole interactions, which are those included in the random-phase approximation (RPA) ${ }^{33}$ and which are known to be the most important interactions for describing single-photon ionization of the rare gases. $^{8-10}$ These particlelike interactions are strongest in the heavier rare gases and have been included in three alternative theoretical treatments for the heavier rare gases: The many-body perturbation theory calculation of the two-photon ionization cross section of argon of Pindzola and Kelly, ${ }^{13}$ the approximate RPA calculation of the two-photon ionization cross sections of neon and argon of Moccia, Rahman, and Rizzo, ${ }^{15}$ and the RPA calculation for the two-photon ionization cross section of xenon of L'Huillier and Wendin. ${ }^{17}$ In addition, we have calculated the effect in argon of certain electron scattering interactions perturbatively (using our transition-matrix results for the atom-photon interactions) because these have been found important in xenon ${ }^{17}$ but have not been treated in previous calculations for argon.
Due to the strength of electron correlations in the heavier rare gases and because of the existence of alternative treatments of two-photon ionization of the outer subshell of the rare gases, ${ }^{13,15,17,34}$ we present our theory explicitly for an atom or ion having an outer $p^{6}$ subshell. Our approach is, however, applicable with minor modifications to $\mathrm{He}\left(\right.$ and $\mathrm{H}^{-}$) as well. Electron correlation effects on the two-photon ionization cross section of He have been treated in the time-dependent Hartree-Fock approximation by Victor ${ }^{12}$ and by Ritchie ${ }^{14}$ as well as by L'Huillier et al. ${ }^{16}$ Two-photon ionization of $\mathrm{H}^{-}$has been treated in the adiabatic hyperspherical approximation by Fink and Zoller ${ }^{18}$ and using discrete basis-set methods by Aymar and Crance. ${ }^{19}$

In Sec. II we present our transition-matrix theory for two-photon ionization of an atom or ion with an outer $p^{6}$ subshell. Our assumptions and approximations are specified and our transition-matrix radial equations are presented explicitly. In Sec. III we discuss the implications of our transition-matrix equations and compare them to those for single-photon ionization. ${ }^{28,30}$ In Sec. IV we discuss certain electron scattering interactions which we have treated perturbatively. In Sec. $\mathrm{V}$ we present our results for the two-photon ionization cross section of the $3 p$ subshell of argon below the one-photon ionization threshold. In Sec. VI we present some conclusions. Preliminary results of this work have been reported elsewhere. ${ }^{35,36}$

\section{TRANSITION-MATRIX METHOD}

The transition-matrix method was developed by Chang and $\mathrm{FanO}^{28}$ specifically to treat electron correlations important for photoionization processes at a level equivalent to that of the random-phase approximation (RPA) or the time-dependent Hartree-Fock method while at the same time maintaining a close connection to the more familiar configuration-interaction picture. Applications ${ }^{29}$ have been made to single photoionization of the outer subshell of argon, a closed-shell atom having a spherically symmetric ground state, as well as to isoelectronic ions and other rare-gas atoms. A graphical method for evaluating the matrix elements needed in the transition-matrix method was developed by Starace and Shahabi. ${ }^{30}$ This graphical method permits a straightforward derivation of the transition-matrix equations for open-shell atoms of arbitrary symmetry. It uncovered as well a new definition of the RPA that is not limited to atoms having a spherically symmetric ground state. ${ }^{30}$ Application has been made to photoionization of atomic chlorine. ${ }^{31,32}$ Each of these earlier works ${ }^{28,30}$ describes in detail the analytical calculations needed to obtain equations for the radial functions which determine the desired transition amplitudes. In our presentation of a transition-matrix method for two-photon ionization of a rare-gas atom, we therefore focus only on the key approximations introduced and the final radial equations obtained and refer the reader to these earlier works ${ }^{28,30}$ for an exposition of the detailed calculational procedures involved. 


\section{A. Implicit summation procedure}

In a two-photon ionization process the transition amplitude $T(\omega)$ for photons of energy $\omega$ between an initial state $i$ and a final state $f$ may be written as

$$
T(\omega)=\sum_{m} \frac{\langle f|D| m\rangle\langle m|D| i\rangle}{\omega+\varepsilon_{i}-\varepsilon_{m}} .
$$

Here $m$ is an intermediate atomic state of energy $\varepsilon_{m}$, and $D$ is the electric dipole operator. Rather than contend directly with an infinite summation over the intermediate states, Dalgarno and Lewis ${ }^{27}$ introduced a differentialequation method for carrying out the summation implicitly. They defined the effective function $\lambda(\omega)$, where

$$
|\lambda(\omega)\rangle=\sum_{m} \frac{|m\rangle\langle m|D| i\rangle}{\omega+\varepsilon_{i}-\varepsilon_{m}},
$$

and showed that $\lambda(\omega)$ may be obtained as the solution of the following inhomogeneous differential equation:

$$
\left(\omega+\varepsilon_{i}-H\right)|\lambda(\omega)\rangle=D|i\rangle .
$$

Then $T(\omega)$ is simply given by

$$
T(\omega)=\langle f|D| \lambda(\omega)\rangle .
$$

The initial and final states, $i$ and $f$, are presumed to be eigenstates of the exact Hamiltonian $H$ with eigenvalues $\varepsilon_{i}$ and $\varepsilon_{f}$, where $\varepsilon_{f}=\varepsilon_{i}+2 \omega$. The main task of any theory of two-photon ionization is then to specify representations for the initial, intermediate, and final states, $i, \lambda$, and $f$, appearing in Eqs. (3) and (4) and to derive solvable equations for any unknown functions appearing in these representations that are needed for the calculation of Eq. (4).

\section{B. First-order transition matrix}

Motivation for a transition-matrix approach stems from the observation that the dipole operator in Eq. (4) is a one-electron operator, i.e.,

$$
D \equiv \widehat{\boldsymbol{\varepsilon}} \cdot \sum_{i=1}^{N} \mathbf{r}_{i}
$$

where $\widehat{\boldsymbol{\varepsilon}}$ is the polarization of the photons and where $\widehat{\mathbf{r}}_{i}$ is the radial coordinate of the $i$ th atomic electron $(1 \leqq i \leqq N)$. Hence, the transition amplitude $T(\omega)$ may be expressed as the one-electron integral, ${ }^{28}$

$$
T(\omega)=\int d \mathbf{r}_{N} \int d \mathbf{r}_{N}^{\prime} \widehat{\boldsymbol{\varepsilon}} \cdot \mathbf{r}_{N} \delta\left(\mathbf{r}_{N}-\mathbf{r}_{N}^{\prime}\right)\left(\mathbf{r}_{N}|\Gamma| \mathbf{r}_{N}^{\prime}\right),
$$

where the first-order transition matrix is defined by ${ }^{28}$

$$
\begin{aligned}
\left(\mathbf{r}_{N}|\Gamma| \mathbf{r}_{N}^{\prime}\right) \equiv & {\left[\prod_{i=1}^{N-1} \int d \mathbf{r}_{i} \int d \mathbf{r}_{i}^{\prime} \delta\left(\mathbf{r}_{i}-\mathbf{r}_{i}^{\prime}\right)\right] } \\
& \times\left\langle\mathbf{r}_{1} \cdots \mathbf{r}_{i} \mid \lambda(\omega)\right\rangle\left\langle f \mid \mathbf{r}_{1}^{\prime} \cdots \mathbf{r}_{N}^{\prime}\right\rangle,
\end{aligned}
$$

i.e., it is the integral over all coordinates but those of the $N$ th electron of the outer product of the intermediate and final states, $|\lambda(\omega)\rangle\langle f|$, where the latter are expressed in coordinate-space representation. In the transition-matrix method an equation for the first-order transition matrix, $\left(\mathbf{r}_{N}|\Gamma| \mathbf{r}_{N}^{\prime}\right)$, is obtained by integrating the following commutator equation for the outer product $|\lambda(\omega)\rangle\langle f|$ over the first $N-1$ electron coordinates:

$[H,|\lambda(\omega)\rangle\langle f|]=-\omega|\lambda(\omega)\rangle\langle f|-D| i\rangle\langle f|$.

A key feature of the transition-matrix approach is that it focuses directly on those electron correlations that are important for calculating the transition amplitude $T(\omega)$ in Eq. (4) and ignores other correlations common to $\lambda(\omega)$ and $f$ which cancel in the commutator equation (8).

\section{State representations}

Those correlations to be considered are restricted by the forms specified for the initial, intermediate, and final states, $i, \lambda(\omega)$, and $f$, in Eq. (8). We emphasize the particle-hole electron correlations known to be important in single-photon ionization processes. We therefore represent the initial, intermediate, and final states as follows:

$$
\begin{aligned}
& |i\rangle=\left|3 p^{6}\left({ }^{1} S\right)\right\rangle+\sum_{\bar{L}, \bar{S}} \sum_{l=2}^{3} b_{l}^{i}(\bar{L} \bar{S})\left|3 p^{4}(\bar{L} \bar{S}) \phi_{l}^{i} \phi_{l}^{i}(\bar{L} \bar{S})\left({ }^{1} S\right)\right\rangle, \\
& |\lambda(\omega)\rangle=\sum_{l_{\lambda}=0,2}\left|3 p^{5} \psi_{l_{\lambda}}^{\lambda}\left({ }^{1} P\right)\right\rangle+\sum_{\bar{L}, \bar{S}} \sum_{L_{p} S_{p}} \sum_{l_{f}=1,3} b_{l_{f}}^{\lambda}\left(\bar{L} \bar{S}, L_{p} S_{p}\right)\left|3 p^{4}(\bar{L} \bar{S}) \phi_{d}^{\lambda} \phi_{l_{f}}^{\lambda}\left(L_{p} S_{p}\right)\left({ }^{1} P\right)\right\rangle, \\
& |f\rangle=\left|3 p^{5} \psi f_{f}\left({ }^{1} L\right)\right\rangle .
\end{aligned}
$$

The ground state $|i\rangle$ is thus represented by an admixture of the normal $3 p^{6}$ configuration plus a sum of doubly excited configurations in which two $3 p$ electrons are virtually excited to a pair of $d$ or $f$ orbitals. The intermediate state $|\lambda(\omega)\rangle$ is represented by an admixture of the expected singly excited state, in which one $3 p$ electron is photoexcited to the $\psi_{l_{\lambda}}^{\lambda}\left(l_{\lambda}=0\right.$ or 2$)$ orbital, and a sum of doubly excited configurations, $\phi_{d}^{\lambda} \phi_{l_{f}}^{\lambda} \quad\left(l_{f}=1,3\right)$, arising from photoexcitation or de-excitation of the doubly excited electron pairs in the ground state: $\phi_{d}^{i} \phi_{d}^{i}$ and $\phi_{f}^{i} \phi_{f}^{i}$. [Note that we ignore intermediate-state pair excitations such as $\phi_{f}^{\lambda} \phi_{g}^{\lambda}$ arising from photoexcitation of $\phi_{f}^{i} \phi_{f}^{i}$ since $\phi_{f}^{\lambda} \phi_{g}^{\lambda}$ cannot be de-excited by the second photon to a final state having a configuration as in $\mathbf{E q}$. (9c).] Our final state is assumed to have only one electron excited out of the $3 p$ subshell. This final-state electron's orbital angular momentum $l_{f}$ is 1 or 3 . Due to angular momentum selection rules for absorption of two photons having the same polarization, the $L=1$ final state is not allowed. 


\section{Two-photon transition amplitude}

The desired transition amplitude $T(\omega)$ is calculated by substituting the states in Eq. (9) in Eq. (4) to obtain

$$
T(\omega)=\left(\psi_{l_{f}}^{f}|r| \psi_{l_{\lambda}}^{\lambda}\right)\left(l_{f}\left\|C^{[1]}\right\| l_{\lambda}\right)[1]^{1 / 2}[L]^{1 / 2}\left\{\begin{array}{ccc}
l_{\lambda} & 1 & 1 \\
L & l_{f} & 1
\end{array}\right\}+\left(\chi_{3 p}|r| \bar{\phi}_{d}^{\lambda}\right)\left(1\left\|C^{[1]}\right\| 2\right)\left[\frac{1}{2}\right]^{1 / 2} .
$$

The reduced angular matrix element in Eq. (10) is defined by

$$
\left(a\left\|C^{[k]}\right\| b\right) \equiv(-1)^{a}[a]^{1 / 2}[b]^{1 / 2}\left(\begin{array}{ccc}
a & k & b \\
0 & 0 & 0
\end{array}\right),
$$

where the six numbers in braces represent a $3-j$ symbol, and $[a] \equiv 2 a+1$. The radial dipole matrix element is defined by

$$
\left(\psi_{a}|r| \psi_{b}\right) \equiv \int_{0}^{\infty} r^{2} d r \psi_{a}^{*}(r) r \psi_{b}(r)
$$

In Eq. (10) we have also defined an effective one-electron correlation function, $\bar{\phi}_{d}^{\lambda}(r)$, as

$$
\begin{aligned}
\left.\bar{\phi}_{d}^{\lambda}(r) \equiv \sum_{l_{f}} \sum_{\bar{L}, \bar{S}} \sum_{L_{p}, S_{p}} b_{l_{f}}^{\lambda}\left(\bar{L} \bar{S}, L_{p} S_{p}\right)\left(3 p^{4}(\bar{L} \bar{S}) \mid\right\} 3 p^{5}\left({ }^{2} P\right)\right)[2]^{1 / 2} \\
\quad \times \phi_{d}^{\lambda}(r)\left(\phi_{l_{f}}^{\lambda} \mid \psi l_{f}^{f}\right) \delta\left(\bar{S}, S_{p}\right)(-1)^{L}[1]\left[L_{p}\right]^{1 / 2}[L]^{1 / 2}\left\{\begin{array}{ccc}
L_{p} & 2 & l_{f} \\
1 & 1 & L \\
\bar{L} & 1 & 1
\end{array}\right\},
\end{aligned}
$$

where $\left(\phi_{l_{f}}^{\lambda} \mid \psi_{l_{f}}^{f}\right)$ is a radial overlap integral, $\delta\left(\bar{S}, S_{p}\right)$ is a $\delta$ function, and the nine numbers in curly brackets comprise a $9-j$ coefficient. Note that $\bar{\phi}_{d}^{\lambda}(r)$ is dependent on the final total orbital angular momentum $L$ as is $\psi_{f}^{f}$, although for simplicity of notation we have not indicated this dependence explicitly.

Examination of Eq. (10) shows that there are two contributions to the desired transition amplitude: a photoexcitation of the intermediate-state electron described by $\psi_{l_{\lambda}}^{\lambda}(r)$ to the final-state electron described by $\psi_{l_{f}}^{f_{f}}(r)$, and a photo de-excitation of an electron described by the effective intermediate-state function $\bar{\phi}_{d}^{\lambda}(r)$ to the $3 p$ subshell, described by the radial function $\chi_{3 p}(r)$. The second contribution to $T(\omega)$ arises from the doubly excited configuration in the intermediate state in Eq. (9b). Note that only one of these doubly excited electrons is de- excited; the other becomes the excited electron in the final state with an amplitude given by the radial overlap integral in Eq. (12).

\section{E. Equations for one-electron radial functions}

Calculation of the desired two-photon transition amplitude in Eq. (10), therefore, requires only that we obtain the one-electron radial functions $\psi_{l_{\lambda}}^{\lambda}(r), \bar{\phi}_{d}^{\lambda}(r), \psi_{l_{f}}(r)$, and $\chi_{3 p}(r)$. We do this by substituting the representations for the initial, intermediate, and final states given in Eq. (9) into the equation of state in Eq. (8), integrating over the first $N-1$ electron spatial and spin coordinates, keeping only particle-hole interactions, and equating to zero the coefficients of certain double-tensor operators as described in detail in Refs. 28 and 30. The resulting differential equation for $\psi_{l_{\lambda}}^{\lambda}(r)$ is

$$
\begin{aligned}
& {\left[-\frac{1}{2} \frac{d^{2}}{d r^{2}}-\frac{Z}{r}+\frac{1}{2} \frac{l(l+1)}{r^{2}}-\left(\omega+\varepsilon_{3 p}\right)\right] \psi_{l}^{\lambda}(r)+\sum_{n_{1} l_{1}}\left[\left(l\left\|V^{0}\left(n_{1} l_{1}, n_{1} l_{1} ; r\right)\right\| l\right) 2 \frac{\left[l_{1}\right]^{1 / 2}}{[l]^{1 / 2}} \psi_{l}^{\lambda}(r)\right.} \\
& \left(n_{1} l_{1} \neq 3 p\right) \\
& \left.+\sum_{k}(-1)^{k+1}\left(l\left\|V^{k}\left(n_{1} l_{1}, \psi_{l}^{\lambda} ; r\right)\right\| l_{1}\right) \frac{1}{[l]} \chi_{n_{1} l_{1}}(r)\right) \\
& +\sum_{k} \sum_{l^{\prime}}\left[\left(l\left\|V^{k}(3 p, 3 p ; r)\right\| l^{\prime}\right)\left[2 \frac{[1]^{1 / 2}}{[l]^{1 / 2}} \delta_{l l^{\prime}} \delta_{k 0}-\left\{\begin{array}{ccc}
1 & 1 & k \\
l^{\prime} & l & 1
\end{array}\right\}\right] \psi_{l^{\prime}}^{\lambda}(r)+\left(l\left\|\boldsymbol{V}^{k}\left(3 p, \psi_{l^{\prime}}^{\lambda} ; r\right)\right\| 1\right)\left[-\frac{2}{3} \delta_{k 1}+\frac{\delta_{l^{\prime} l}}{[l]}\right] \chi_{3 p}(r)\right] \\
& =+2^{1 / 2}\left(l\left\|C^{[1]}\right\| 1\right) \chi_{3 p}(r) r+\sum_{\bar{L}, \bar{S}}(2 \times 3 \times 5)^{1 / 2}\left(3 p^{5}\left({ }^{2} P\right)\left\{\mid 3 p^{4}(\bar{L} \bar{S})\right) b_{l}^{i}(\bar{L} \bar{S})\left(1\left\|C^{[1]}\right\| l\right)\right. \\
& \times\left(\chi_{3 p}|r| \phi_{l}^{i}\right)(-1)^{\bar{L}}\left\{\begin{array}{ccc}
l & l & \bar{L} \\
1 & 1 & 1
\end{array}\right\} \phi_{l}^{i}(r) .
\end{aligned}
$$


The differential equation for $\bar{\phi}_{d}^{\lambda}(r)$ is

$$
\begin{aligned}
& \left(-\frac{1}{2} \frac{d^{2}}{d r^{2}}-\frac{Z}{r}+\frac{1}{2} \frac{2(2+1)}{r^{2}}+\left(\omega-\varepsilon_{3 p}\right)\right) \bar{\phi}_{d}^{\lambda}(r)+\sum_{\substack{n_{1}, l_{1} \\
\left(n_{1} l_{1} \neq 3 p\right)}}\left\{\left(2\left\|V^{0}\left(n_{1} l_{1}, n_{1} l_{1} ; r\right)\right\| 2\right) 2 \frac{\left[l_{1}\right]^{1 / 2}}{[2]^{1 / 2}} \bar{\phi}_{d}^{\lambda}(r)\right. \\
& \left.+\sum_{k}\left(2\left\|V^{k}\left(n_{1} l_{1}, \bar{\phi}_{d}^{\lambda} ; r\right)\right\| l_{1}\right) \frac{(-1)^{1+k}}{[2]} \chi_{n_{1} l_{1}}(r)\right) \\
& +\sum_{k}\left[\left(2\left\|V^{k}(3 p, 3 p ; r)\right\| 2\right)\left[2 \frac{[1]^{1 / 2}}{[2]^{1 / 2}} \delta_{k 0}-\left\{\begin{array}{ccc}
k & 2 & 2 \\
1 & 1 & 1
\end{array}\right\}\right) \bar{\phi}_{d}^{\lambda}(r)+\left(2\left\|V^{k}\left(3 p, \bar{\phi}_{d}^{\lambda} ; r\right)\right\| 1\right)\left[\frac{1}{5}-\frac{2}{3} \delta_{k 1}\right] \chi_{3 p}(r)\right] \\
& \left.=-\sum_{\bar{L}, \bar{S}} b_{d}^{i}(\bar{L} \bar{S})(3 \times 5)^{1 / 2}\left(3 p^{4}(\bar{L} \bar{S}) \mid\right\} 3 p^{5}\left({ }^{2} P\right)\right)\left(l_{f}\left\|C^{[1]}\right\| 2\right)[1]^{1 / 2}[L]^{1 / 2}\left(\phi_{d}^{i}|r| \psi f_{f}\right) \phi_{d}^{i}(r)(-1)^{\bar{L}+1} \\
& \times\left\{\begin{array}{ccc}
2 & 2 & \bar{L} \\
1 & 1 & 1
\end{array}\right\}\left\{\begin{array}{ccc}
1 & 2 & 1 \\
1 & L & l_{f}
\end{array}\right\} \\
& \left.-\sum_{\bar{L}, \bar{S}} b_{l_{f}}^{i}(\bar{L} \bar{S})(3 \times 5)^{1 / 2}\left(3 p^{4}(\bar{L} \bar{S}) \mid\right\} 3 p^{5}\left({ }^{2} P\right)\right)\left(2\left\|C^{[1]}\right\| l_{f}\right) \\
& \times[1]^{1 / 2}[L]^{1 / 2}\left(\phi_{l_{f}}^{i} \mid \psi_{f_{f}}\right) \phi_{l_{f}}^{i}(r) r(-1)^{1+L+\bar{L}}\left\{\begin{array}{ccc}
l_{f} & l_{f} & \bar{L} \\
1 & 1 & L
\end{array}\right\}\left\{\begin{array}{ccc}
1 & L & 1 \\
1 & 2 & l_{f}
\end{array}\right\} .
\end{aligned}
$$

In Eqs. (13) and (14) the following reduced matrix element resulting from the Coulomb interaction has been defined $^{28}$

$$
\begin{aligned}
& \left(l_{2}\left\|V^{k}\left(n_{1} l_{1}, n_{3} l_{3} ; r\right)\right\| l_{4}\right) \\
& \equiv \int_{0}^{\infty} d r \frac{r_{<}^{k}}{r_{>}^{k+1}} P_{n_{1} l_{1}}(r) P_{n_{3} l_{3}}(r) \\
& \quad \times\left(l_{1}\left\|C^{[k]}\right\| l_{3}\right)\left(l_{2}\left\|C^{[k]}\right\| l_{4}\right) .
\end{aligned}
$$

Our expression in Eq. (10) for the transition amplitude for two-photon ionization of the outer $p^{6}$ subshell of a rare-gas atom or ion together with Eqs. (13) and (14) for the two intermediate-state radial functions, $\psi_{l}^{\lambda}(r)$ and $\bar{\phi}_{d}^{\lambda}(r)$, that are required for its evaluation are the main formal results of this paper. We discuss the implications of these results in Sec. III. Here we indicate how the other radial functions needed to calculate Eq. (10) and to solve Eqs. (13) and (14) are obtained.

Firstly, as is the case in single-photon ionization, we assume that the radial functions $\chi_{n l}(r)$ for the bound $n l$ electrons are obtained from a Hartree-Fock (HF) calculation for the ${ }^{1} S$ ground state of the atom or ion.

Secondly, the final-state radial function $\psi_{f}(r)$ is obtained from an $L S$-dependent $\mathrm{HF}$ calculation in the field of the unrelaxed ion having an outer $p^{5}$ subshell. The radial equation is similar in form to that for $\psi_{l_{\lambda}}^{\lambda}(r)$ in Eq. (13) except for the absence of source terms. Unlike the case of single-photon ionization we find that the final-state radial function $\psi f_{f}$ does not couple to the intermediatestate correlation function $\bar{\phi}_{d}^{\lambda}(r)$.

Lastly, the initial-state correlation functions $\phi_{l}^{i}(l=2,3)$ may be obtained using the multiconfiguration $\mathrm{HF}$ code of Froese Fischer. ${ }^{37}$ The use of an average function, $\phi_{l}^{i}$, instead of functions dependent on the term level, $\bar{L} \bar{S}$, of the $p^{4}$ core [cf. Eq. (9a)] has been found to be a good approximation in single-photon ionization studies of rare gases. ${ }^{38}$

\section{DISCUSSION}

We have shown in this paper how those electron correlations found to be most important in single-photon ionization,,$^{8-10}$ the particle-hole interactions, may be included in a calculation of two-photon ionization processes using a transition-matrix approach. A key feature of our approach, as shown in the representation of states in Eq. (9), is the inclusion of excited virtual pairs of electrons in both initial and intermediate states. The two-photon transition amplitude in Eq. (10) is found to have two terms, a photoexcitation term, and a photo de-excitation term, as in the case of single-photon ionization. Unlike the case of single-photon ionization, the transition-matrix equations for the radial functions involved are uncoupled. In addition, there are source terms arising from dipole excitation of the initial state. We discuss each of these aspects of our results in turn.

\section{A. Comparison with single-photon transition-matrix equations}

Our radial equations (13) and (14) are identical to the single-photon transition-matrix equations ${ }^{39}$ except for, firstly, the absence of coupling between $\psi_{l_{f}}^{f}(r)$ and $\bar{\phi}_{d}^{\lambda}(r)$, and, secondly, the presence of the source terms arising from the implicit summation over intermediate states [cf. Eq. (3)]. The lack of coupling in the two-photon case arises from the different tensorial properties of the second 
photon's excitation and de-excitation transitions. To understand the absence of coupling in the two-photon case, consider how the coupling comes about in the one-photon case.

In single-photon ionization of argon, for example, the following direct photoexcitation process,

$$
\operatorname{Ar} 3 p^{6}\left({ }^{1} S\right)+\gamma \rightarrow \operatorname{Ar}^{+} 3 p^{5} \psi f_{f}\left({ }^{1} P\right),
$$

has in the case of $l_{f}=2$ the same tensorial properties as the following photo de-excitation process:

$$
\begin{gathered}
\operatorname{Ar}^{* *} 3 p^{4} \phi_{d}^{i} \phi_{d}^{i}\left({ }^{1} S\right)+\gamma \\
\underset{\substack{\text { Coulomb } \\
\text { interaction }}}{\longrightarrow} \operatorname{Ar}^{* * *} 3 p^{3} \psi_{d} \phi_{d}^{i} \phi^{5} \phi_{d}^{i}\left({ }^{i}\left({ }^{1} P\right)\right.
\end{gathered}
$$

That is, both (16a) (for $l_{f}=2$ ) and (16b) represent $p \rightarrow d$ transitions: Equation (16a) describes excitation of a $3 p$ electron directly to the continuum state represented by the photoelectron's $d$-orbital wave function, $\psi f_{f}=2$, whereas Eq. (16b) describes the excitation of a $3 p$ electron to some excited $d$ orbital, represented by $\psi_{d}$, followed by a Coulomb interaction which de-excites the electron in $\psi_{d}$ and one of the electrons represented by the correlation function $\phi_{d}^{i}$ to the $3 p$ subshell leaving a remaining $\phi_{d}^{i}$ electron to go off with the appropriate photon energy. In the transition matrix method ${ }^{28,30}$ this common $p \rightarrow d$ transition in the one-photon ionization case leads to a coupling between $\psi_{f}(r)$ for $l_{f}=2$ and $\phi_{d}^{i}(r)$.

In the two-photon ionization case, however, the direct photoexcitation from the intermediate state to the final state is of the form

$$
\mathrm{Ar}^{+} 3 p^{5} \psi_{l_{\lambda}}^{\lambda}\left({ }^{1} P\right)+\gamma \rightarrow \mathrm{Ar}^{+} 3 p^{5} \psi_{l_{f}}^{f}\left({ }^{1} L\right)
$$

and the de-excitation process analogous to (16b) is

$$
\begin{aligned}
& \operatorname{Ar}^{* *} 3 p^{4} \phi_{d}^{\lambda} \phi_{l_{f}}^{\lambda}\left({ }^{1} P\right)+\gamma \rightarrow \operatorname{Ar}^{* * *} 3 p^{3} \psi_{d} \phi_{d}^{\lambda} \phi_{l_{f}}^{\lambda}\left({ }^{1} L\right) \\
& \underset{\substack{\text { Coulomb } \\
\text { interaction }}}{\longrightarrow} \operatorname{Ar}^{+} 3 p^{5} \phi_{l_{f}}^{\lambda}\left({ }^{1} L\right) .
\end{aligned}
$$

Since $l_{\lambda}=0$ or 2 and $l_{f}=1$ or 3 we see that the $l_{\lambda} \rightarrow l_{f}$ transition in Eq. (17a) has different tensorial properties from the $p \rightarrow d$ transition in Eq. (17b). For this reason $\psi f_{f}$ is uncoupled from the effective intermediate-state correlation function $\bar{\phi}_{d}^{\lambda}$ defined in Eq. (12) and hence $\psi f_{f}$ may be obtained from a HF or close-coupling calculation (the latter including coupling between the $l_{f}=3$ and $l_{f}=1$ channels).

\section{B. Source terms}

The radial functions $\psi_{l}^{\lambda}(r)$ and $\bar{\phi}_{l}^{\lambda}(r)$ defined in Eqs. (13) and (14) arise due to the source terms on the righthand sides of these equations. On the left-hand side of each equation are simply $\mathrm{HF}$ or close-coupling radial differential operators. It is therefore of interest to examine the nature of each of the source terms on the righthand sides of Eqs. (13) and (14).

The first term on the right in Eq. (13), proportional to $r \chi_{3 p}(r)$, is the usual source term for populating the intermediate excited state $\psi_{l}^{\lambda}(r)$ : namely, a dipole transition of a $3 p$-subshell electron to a state of angular momentum $l$. This term stems from the usual $3 p^{6}$ configuration of the argon ground state [cf. the first term of Eq. (9a)]. The second term on the right of Eq. (13), proportional to $\left(\chi_{3 p}|r| \phi_{l}^{i}\right) \phi_{l}^{i}(r)$, arises from the virtual doubly excited states in the argon ground state [cf. the second term of Eq. (9a)]. In this case the first photon de-excites one of the doubly excited electrons, described by $\phi_{l}^{i}$, to the $3 p$ subshell with an amplitude proportional to $\left(\chi_{3 p}|r| \phi_{l}^{i}\right)$. The other doubly excited electron, also described by $\phi_{l}^{i}$, then is left to contribute to the amplitude $\psi_{l}^{\lambda}(r)$. Note that since $\psi_{l}^{\lambda}(r)$ can only have $l=0$ or 2 (by dipole selection rules) and since $\phi_{l}^{i}(r)$ is assumed to have $l$ values of 2 or 3 , it is obvious that only $\phi_{d}^{i}(r)$ contributes to $\psi_{d}^{\lambda} ; \phi_{f}^{i}(r)$ plays no role here.

The intermediate-state correlation function $\bar{\phi}_{d}^{\lambda}(r)$, defined by Eqs. (9b), (12), and (14), also has two source terms given on the right of Eq. (14). Both of these source terms originate with the correlated part of the initial state in Eq. (9a). The first source term, proportional to $\left(\phi_{d}^{i}|r| \psi_{f}\right) \phi_{d}^{i}(r)$, arises from an electric dipole excitation of the virtually excited ground-state pair $\phi_{d}^{i} \phi_{d}^{i}$ to $\psi_{f}^{f} \phi_{d}^{i}$. The second photon will then de-excite $\phi_{d}^{i}$ back to the $3 p$ subshell leaving only a single electron in the excited state, $\psi f_{f}$. This de-excitation by the second photon is described by the second term in the two-photon transition amplitude in Eq. (10).

$\bar{\phi}_{d}^{\lambda}(r)$ is also populated by the second source term in Eq. (14), which is proportional to $\left(\phi_{l_{f}}^{i} \mid \psi f_{f}\right) \phi_{l_{f}}^{i}(r) r$. Since $\phi_{l_{f}}^{i}$ is assumed to have the values $l_{f}=2,3$ and since $\psi_{l_{f}}$ can only have the values 1 or 3 , clearly only $\phi_{f}^{i} \phi_{f}^{i}$ contributes here. In fact this is the only contribution that $\phi_{f}^{i} \phi_{f}^{i}$ makes in our treatment. This source term describes one of the $\phi_{f}^{i}$ electrons overlapping with the final-state wave function $\psi_{f}^{f}$ while the other $\phi_{f}^{i}$ electron is de-excited first to a $d$ orbital, which contributes in Eq. (14) to $\bar{\phi}_{d}^{\lambda}(r)$, and then to the $3 p$ subshell, as in the second term of the twophoton transition amplitude in Eq. (10).

\section{Role of virtual doubly excited states}

While it is well established that virtual doubly excited states are essential to a theoretical description of singlephoton ionization processes, ${ }^{8-10}$ what role they play in multiphoton processes is still an open question. Are they only necessary in describing absorption of the first photon? Or, must they be considered in every intermediate state of a multiphoton process? Our formulation is designed to answer this crucial question.

If the contribution of $\bar{\phi}_{d}^{\lambda}$ to the two-photon transition amplitude [cf. the second term in Eq. (10)] is negligible, then a theoretical description of two or more photon ionization processes including the important particle-hole electron correlations will be straightforward. Both of the electron correlations that are important in single-photon ionization from the ground state are already included in Eq. (13) for the function $\psi_{l}^{\lambda}(r)$. Extension of our treatment of two-photon ionization processes to multiphoton ionization processes involving three or more photons will then involve merely HF-type iterations of the Dalgarno- 
Lewis differential Eq. (3) once the first intermediate state is calculated according to Eq. (13).

If, on the other hand, the contribution of $\bar{\phi}_{d}^{\lambda}$ to the two-photon transition amplitude in Eq. (10) is significant, then this will imply that virtual electron pairs must be included theoretically in the intermediate states of a multiphoton process. Extension of our two-photon treatment, presented in this paper, to three or more photon processes would be nontrivial. In particular, the second-order transition matrix ${ }^{28}$ would need to be introduced to describe the propagation of the virtual electron pairs from one intermediate state to another. In short, Eq. (14) would have to be generalized to a pair equation; one could no longer get by with a one-electron function such as $\bar{\phi}_{d}^{\lambda}(r)$ to represent a pair of virtually excited electrons.

Numerical calculations for two-photon ionization of argon using the theory presented here are presented below. These calculations answer the theoretical questions posed here on the role of virtual pairs of electrons in multiphoton ionization.

\section{ELECTRON SCATTERING INTERACTIONS}

Until very recently, all calculations for two-photon one-electron ionization processes restricted their treatment of electron correlations to those in which only one of the two photons interacts with the target and the other photon interacts with one of the electrons excited from the target. Our transition-matrix approach, described above, also employs this restriction. (Our approach can treat a more general class of interactions, but the calculation of interactions between atomic states having several electrons excited from the target quickly becomes rather complex.) L'Huillier et al. ${ }^{16,17}$ approximately treated in addition to these interactions a set of electron correlations in which each photon excites an electron from the unexcited atomic target. The electrons then interact in such a way that one electron is de-excited back to the core and the other goes off as the single observable photoelectron. These interactions were found by L'Huillier and Wendin ${ }^{17}$ to reduce the two-photon single-ionization cross section of atomic xenon by a large fraction in the region below the singlephoton ionization threshold. In the region above the single-photon ionization threshold, these interactions were found to increase the cross sections by an order of magnitude.

These interactions are specified exactly (in lowest order) by the many-body perturbation theory (MBPT) diagrams shown in Figs. 1(a) and 1(b); a more informal "picture" of these interactions is provided in Figs. $1\left(a^{\prime}\right)$ and $1\left(b^{\prime}\right)$ for those who are not conversant with MBPT. Each of the diagrams in Fig. 1 describes a particular path by which the following general process occurs:

$$
X+2 \gamma \rightarrow X^{+}\left(n_{0} l_{0}^{-1}\right)+e^{-},
$$

i.e., the process in which two photons ionize a single electron from the $n_{0} l_{0}$ subshell of atom $X$. In Figs. 1(a) and $1\left(a^{\prime}\right)$ the photoelectron having kinetic energy $\varepsilon$ and orbital angular momentum $l$ that is ionized by the first photon from the atom's $n_{0} l_{0}$ subshell scatters from the residual ion core. By means of the Coulomb interaction, it excites

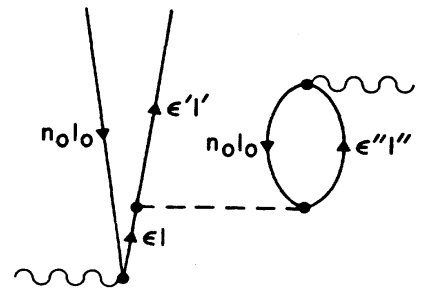

(a)

(b)

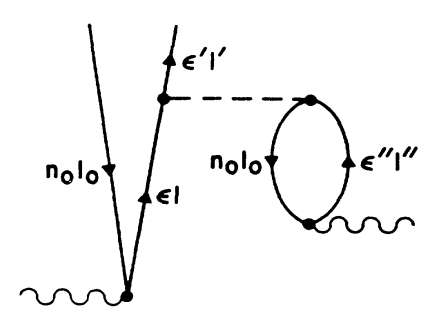

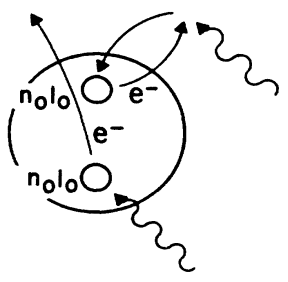

(a')

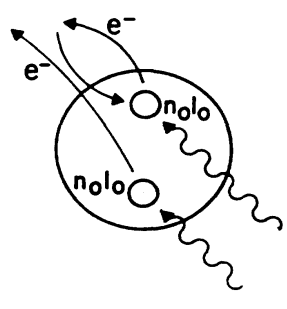

(b')
FIG. 1. Electron scattering interactions relevant to twophoton ionization processes. See text for description. Manybody-perturbation-theory diagrams are shown in (a) and (b). Corresponding schematic scattering diagrams are shown in $\left(a^{\prime}\right)$ and $\left(b^{\prime}\right)$.

a second $n_{0} l_{0}$ subshell electron to the state $\varepsilon^{\prime \prime} l^{\prime \prime}$; as a result of this interaction, the first electron is scattered into the $\varepsilon^{\prime} l^{\prime}$ state. The second photon then de-excites the $\varepsilon^{\prime \prime} l^{\prime \prime}$ electron back to the $n_{0} l_{0}$ subshell, leaving the single $\varepsilon^{\prime} l^{\prime}$ electron to go off.

In Figs. $1(b)$ and $1\left(b^{\prime}\right)$ on the other hand, each photon excites an $n_{0} l_{0}$ subshell electron (to the states $\varepsilon l$ and $\left.\varepsilon^{\prime \prime} l^{\prime \prime}\right)$. These two excited electrons then interact via the Coulomb interaction in such a way that the $\varepsilon l$ electron is scattered into the state $\varepsilon^{\prime} l^{\prime}$ while the $\varepsilon^{\prime \prime} l^{\prime \prime}$ electron is deexcited back to the $n_{0} l_{0}$ core. An additional diagram that must be considered, but which is not shown in Fig. 1, is very similar to that in Fig. $1(\mathrm{~b})$ but differs in that the $\varepsilon^{\prime \prime} l$ l" electron is excited before the $\varepsilon l$ electron, where time in these diagrams is directed from bottom to top.

It is intuitively clear why the interactions in Fig. 1 turn out to be important. One knows that the single-photon electric dipole transition from the outer $p^{6}$ subshell of rare-gas atoms is very strong, particularly above the single-photon ionization threshold. ${ }^{8-10}$ The diagrams in Fig. 1 introduce this interaction twice, once for each photon. Roughly speaking, this transition amplitude is stronger for an $n_{0} l_{0}$ electron than for an excited electron by a factor corresponding to the occupation number of the $n_{0} l_{0}$ subshell; however, potential barrier effects important for the transition $n_{0} l_{0} \rightarrow \varepsilon\left(l_{0}+1\right)$ come into play as well.

Exact evaluation of the interactions in Fig. 1 by the transition-matrix approach is complex. The doubly excited intermediate states introduce complicated angular momentum algebra and summations over coefficients of fractional parentage. L'Huillier et al. ${ }^{16,17}$ treat the interactions in Fig. 1 by means of the random-phase approximation (RPA): Each interaction of a photon with 
the $n_{0} l_{0}$ subshell is treated as if it were a transition of the type, $\quad n_{0} l_{0}^{4 l_{0}+2}\left({ }^{1} S\right)+\gamma \rightarrow n_{0} l_{0}^{4 l_{0}+1} \varepsilon l\left({ }^{1} P\right)$, i.e., a singlephoton ionization process from the atomic ground configuration, in which the transition amplitude used is the RPA amplitude.

Because of the significance of the electron scattering interactions, we include their effect on our transition ampli- tude in the same approximate way as done in the work of L'Huillier et al. ${ }^{16,17}$ That is, we use our result for $\psi_{l}^{\lambda}(r)$ in Eq. (13) to describe the effective intermediate state [defined in Eqs. (2) and (9b)] resulting from the photon's interaction with the atom.

In this way, the transition amplitude in Eq. (10) is modified to

$$
\begin{aligned}
T(\omega)= & \left(l_{f}\left\|C^{[1]}\right\| l_{\lambda}\right)[1]^{1 / 2}[L]^{1 / 2}\left\{\begin{array}{ccc}
l_{\lambda} & 1 & 1 \\
L & l_{f} & 1
\end{array}\right\} \\
& \times\left[\left(\psi_{l_{f}}^{f}|r| \psi_{l_{\lambda}}^{\lambda}\left(\omega+\varepsilon_{3 p}\right)\right)-3^{-1 / 2} \sum_{l_{\lambda}^{\prime}}\left(1\left\|C^{[1]}\right\| l_{\lambda}^{\prime}\right) R^{1}\left(\psi_{l_{f}}^{f}, \psi_{l_{\lambda}}^{\lambda}\left(\omega+\varepsilon_{3 p}\right) ; \chi_{3 p}, \psi_{l_{\lambda}^{\prime}}^{\lambda}\left(\omega+\varepsilon_{3 p}\right)\right)\right. \\
& \left.-3^{-1 / 2} \sum_{l_{\lambda}^{\prime}}\left(l_{\lambda}^{\prime}\left\|C^{[1]}\right\| 1\right) R^{1}\left(\psi_{f}^{f}, \psi_{l_{\lambda}}^{\lambda}\left(\omega+\varepsilon_{3 p}\right) ; \psi_{l_{\lambda}^{\prime}}^{\lambda}\left(\varepsilon_{3 p}-\omega\right), \chi_{3 p}\right)\right]+\left(\chi_{3 p}|r| \bar{\phi}_{d}^{\lambda}\right)\left(1\left\|C^{[1]}\right\| 2\right)\left[\frac{1}{2}\right]^{1 / 2}
\end{aligned}
$$

In Eq. (19), the third term in large parentheses describes the interaction in Figs. 1(a) and $1\left(a^{\prime}\right)$ while the second term in large parentheses describes the interaction shown in Figs. $1(b)$ and $1\left(b^{\prime}\right)$ as well as the corresponding interaction in which the time ordering of the photons is reversed. Also in Eq. (19), radial Slater integrals are indicated by $R^{1}$, and the energy at which each $\psi^{\lambda}$ is calculated is indicated in parenthesis [cf. Eq. (13)].

Note that interactions of higher order than shown in Fig. 1 are included implicitly in our expression for the transition amplitude in Eq. (19). Due to the second source term on the right of Eq. (13) above, the effects of electron pairs virtually excited out of the ground state are included also in describing the photon-atom interaction. Such higher-order effects are included also in Refs. 16 and 17 , where they are included as part of the "screened interaction" described there.

\section{APPLICATION TO TWO-PHOTON IONIZATION OF THE Ar $3 p$ SUBSHELL}

We are concerned with the calculation of the cross sections for each of the following transitions:

$$
\begin{aligned}
\operatorname{Ar} 3 p\left({ }^{6} S\right)+2 \gamma & \rightarrow \mathrm{Ar}^{+} 3 p 5\left({ }^{2} P\right) \varepsilon f\left({ }^{1} D\right) \\
& \rightarrow \mathrm{Ar}^{+} 3 p 5\left({ }^{2} P\right) \varepsilon p\left({ }^{1} D\right) \\
& \rightarrow \mathrm{Ar}^{+} 3 p 5\left({ }^{2} P\right) \varepsilon p\left({ }^{1} S\right)
\end{aligned}
$$

We present in turn some numerical details of our calculations, discussion of our results, and comparisons with results of other authors.

\section{A. Numerical details}

Our initial state is represented as a superposition of the frozen core Hartree-Fock (HF) ground-state configuration, $3 p^{6}\left({ }^{1} S\right)$, and configurations having pairs of $d$ and $f$ electrons excited out of the ground-state configuration. As shown by Froese Fischer, ${ }^{37}$ such excited pairs of electrons may be well represented by a diagonal expansion of the form $3 d^{2}, 4 d^{2}, \ldots, 4 f^{2}, 5 f^{2}, \ldots$. Furthermore, Swanson and Armstrong ${ }^{38}$ have found that in the case of argon these expansions are so rapidly convergent that only the leading terms need to be included. We have therefore included only the $3 d^{2}$ and $4 f^{2}$ excited pairs in our representation for the initial state. We have used the MCHF computer program of Froese Fischer ${ }^{40}$ to calculate firstly the Ar $3 p^{6}\left({ }^{1} S\right)$ single-configuration ground-state wave functions. These were then used as "frozen" input orbitals to a multiconfiguration calculation that mixed the $3 p^{6}$ configuration with configurations of the type $3 p^{4} 3 d^{2}$ and $3 p^{4} 4 f^{2}$. The calculated weights of each of these configurations are given in Table I. Note that only a single $3 d$ orbital wave function and a single $4 f$ orbital wave function were calculated, i.e., as in Ref. 38, the term dependence of these orbitals was ignored.

The final-state wave function for the photoelectron in each of the configurations on the right in Eq. (20) was calculated in the $V^{N-1}(L S)$ potential ${ }^{41}$ for the ion. We employed the frozen core approximation, i.e., the ionic oneelectron orbitals of the $\mathrm{Ar}^{+} 3 p^{5}\left({ }^{2} \mathrm{P}\right)$ configuration were the same as those calculated for the $\operatorname{Ar} 3 p^{6}\left({ }^{1} S\right)$ initial configuration. Orthogonality of the continuum $p$-electron wave functions to the bound $2 p$ and $3 p$ wave functions was assured by calculating both the bound and continuum orbitals in the same Hermitian potential, as described in Refs. 42-44 and as illustrated in detail for photoionization of atomic chlorine in Ref. 45. Each of our continuum wave functions was energy normalized at large radial distances:

$$
\psi f_{f}(k, r)=\left(\frac{2}{\pi \zeta\left(l_{f}, k, r\right)}\right)^{1 / 2} \sin \left[\theta\left(l_{f}, k, r\right)+\delta\right],
$$

where

$$
\begin{aligned}
& \theta\left(l_{f}, k, r\right) \underset{r \rightarrow \infty}{\rightarrow} k r-\frac{1}{2} \pi l_{f}+k^{-1} \ln (2 k r) \\
&+\arg \Gamma\left(l_{f}+1-i / k\right), \\
& \zeta\left(l_{f}, k, r\right) \underset{r \rightarrow \infty}{\rightarrow} k .
\end{aligned}
$$


TABLE I. Weights of initial-state configurations.

\begin{tabular}{lr}
\hline \hline Configuration & Weight $^{\mathrm{a}}$ \\
\hline Ar $3 p^{6}\left({ }^{1} S\right)$ & 0.9776 \\
Ar $3 p^{4}\left({ }^{1} S\right) 3 d^{2}\left({ }^{1} S\right)$ & 0.1101 \\
Ar $3 p^{4}\left({ }^{1} D\right) 3 d^{2}\left({ }^{1} S\right)$ & 0.1200 \\
Ar $3 p^{4}\left({ }^{3} P\right) 3 d^{2}\left({ }^{1} S\right)$ & 0.1268 \\
Ar $3 p^{4}\left({ }^{1} S\right) 4 f^{2}\left({ }^{1} S\right)$ & -0.0237 \\
Ar $3 p^{4}\left({ }^{1} D\right) 4 f^{2}\left({ }^{1} S\right)$ & -0.0233 \\
Ar $3 p^{4}\left({ }^{3} P\right) 4 f^{2}\left({ }^{1} S\right)$ & -0.0261 \\
\hline \hline
\end{tabular}

${ }^{a}$ Calculated using the MCHF computer program of Froese Fischer (cf. Ref. 40).

In Eq. (21), $k^{2} / 2$ is the kinetic energy of the photoelectron and $\delta$ is the phase shift with respect to a Coulomb wave. The phase function $\theta\left(l_{f}, k, r\right)$ and the amplitude function $\zeta\left(l_{f}, k, r\right)$ are calculated at large but finite $r$ using the procedure of Martins. ${ }^{46}$ In our calculations we have ignored the final-state interchannel coupling between the $f^{1} D$ and $p{ }^{1} D$ channels.

The intermediate-state functions, $\psi_{l_{\lambda}}^{\lambda}$ and $\bar{\phi}_{d}^{\lambda}$ [cf. Eq. (12)], which are needed to calculate the transition amplitudes in Eqs. (10) and (19), were obtained as the solutions of Eqs. (13) and (14). Each of the source terms on the right in these two equations was calculated using the one-electron orbital wave functions calculated for the initial and final configurations. Since we have restricted our calculations to the region below the one-photon ionization threshold, both $\psi_{l_{f}}^{\lambda}(r)$ and $\bar{\phi}_{d}^{\lambda}(r)$ satisfy boundstate boundary conditions at large $r$. In solving Eqs. (13) and (14) we have used the $\mathrm{HF}$ value for the $3 p$-orbital energy, $\varepsilon_{3 p}$, i.e., $\varepsilon_{3 p}^{\mathrm{HF}}=-0.5910$ a.u., where 1 a.u. $=$ $27.2108 \mathrm{eV}$.

\section{B. Results and discussion}

\section{Generalized two-photon cross-section results for individual channels}

In lowest-order perturbation theory the $N$-photon ionization rate, $W$, is given by ${ }^{47}$

$$
W=\sigma_{N} I^{N} \text {. }
$$

Here $\sigma_{N}$ is a generalized $N$-photon cross section dependent only on properties of the atomic or molecular target and on the polarization of the incident light, and $I$ is the intensity of the laser field. $W$ is usually measured in units of ions/sec, $\sigma_{N}$ in units of $\mathrm{cm}^{2 N} \mathrm{sec}^{N-1}$, and $I$ in units of photons $/\left(\mathrm{cm}^{2} \mathrm{sec}\right)$. For two-photon ionization, the generalized cross section in atomic units (i.e., $e=\hbar=m=1$ ) is

$$
\sigma_{2}^{q}=\frac{8 \pi^{3} \omega^{2}}{c^{2}}|T|^{2} f_{q} \text { (a.u. ) . }
$$

Here $\omega$ is the photon energy, $c$ is the speed of light, $T$ is the reduced transition-matrix element [cf. Eqs. (10) and (19) ], and $f_{q}$ is a geometrical factor dependent on the polarization $q$ of the photons and on the final-state term level. Table II gives the values of $f_{q}$ for the cases of interest in this paper. Conversion of Eq. (23) to the usual units,
TABLE II. Values for $f_{q}$ [cf. Eq. (23)] for linearly polarized $(q=0)$ and circularly polarized $(q= \pm 1)$ photons.

\begin{tabular}{cll}
\hline \hline & $\begin{array}{l}\text { Final-state } \\
\text { term level }\end{array}$ & $f_{q}$ \\
\hline 0 & ${ }^{1} S$ & $\frac{1}{9}$ \\
0 & ${ }^{1} D$ & $\frac{2}{45}$ \\
\pm 1 & ${ }^{1} S$ & 0 \\
\pm 1 & ${ }^{1} D$ & $\frac{1}{15}$ \\
\hline
\end{tabular}

$\mathrm{cm}^{4} \mathrm{sec}$, requires the conversion of the dimensions, $L^{4} T$, from a.u. to cgs units:

$$
L^{4} T \text { (a.u.) }=1.8967 \times 10^{-50} \mathrm{~cm}^{4} \mathrm{sec} .
$$

We present our length form results for the generalized two-photon cross section for each of the three transitions in Eq. (20) in the case of linearly polarized photons in Figs. 2-4. Our results are shown in four levels of approximation, which we discuss in turn. In the Hartree-Fock

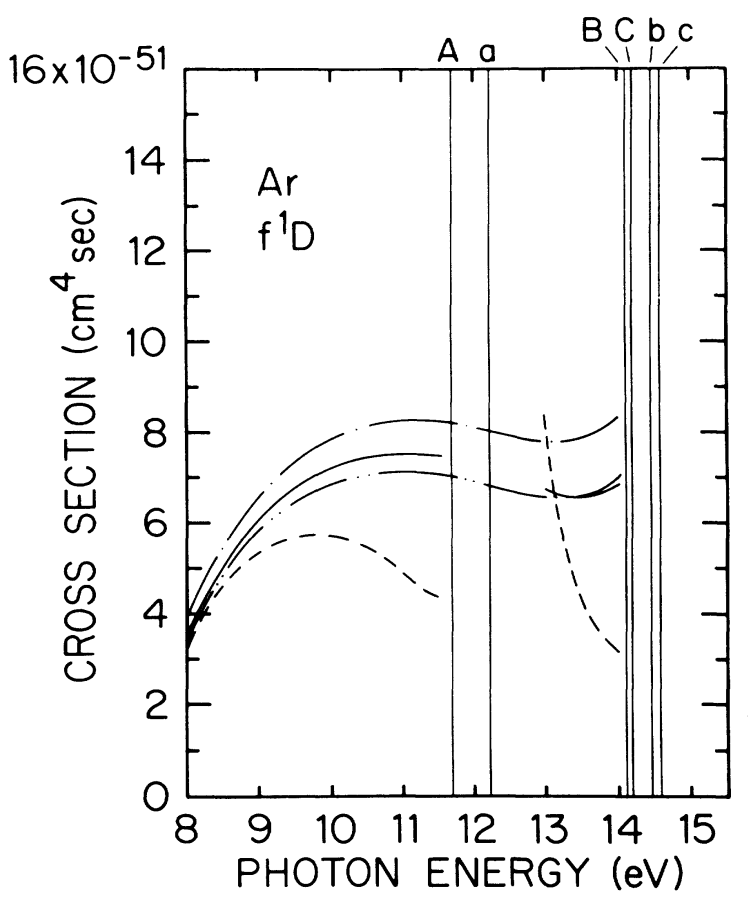

FIG. 2. Generalized two-photon cross section (length form) for the transition $\operatorname{Ar} 3 p^{6}\left({ }^{1} S\right)+2 \gamma \rightarrow \mathrm{Ar}^{+} 3 p^{5} \varepsilon f\left({ }^{1} D\right)$ for photon energies below the single-ionization threshold. The locations of the $\operatorname{Ar} 3 p^{5} 4 s\left({ }^{1} P\right)$, Ar $3 p^{5} 5 s\left({ }^{1} P\right)$, and $\operatorname{Ar} 3 p^{5} 3 d\left({ }^{1} P\right)$ intermediatestate resonances are indicated by the vertical lines, which are labelled, respectively, by $A, B$, and $C$ for the experimental resonance energies and $a, b$, and $c$ for the Hartree-Fock (HF) resonance energies. Our results are given in four different approximations, discussed in the text, which are identified as follows: dash-dot curve, HF results; dash-double-dot curve, HF plus ground-state correlations (GSC); solid curve, HF plus GSC plus intermediate-state interchannel coupling (IIC); dashed curve, $\mathbf{H F}$ plus GSC plus IIC plus electron-scattering interactions (ESI). 


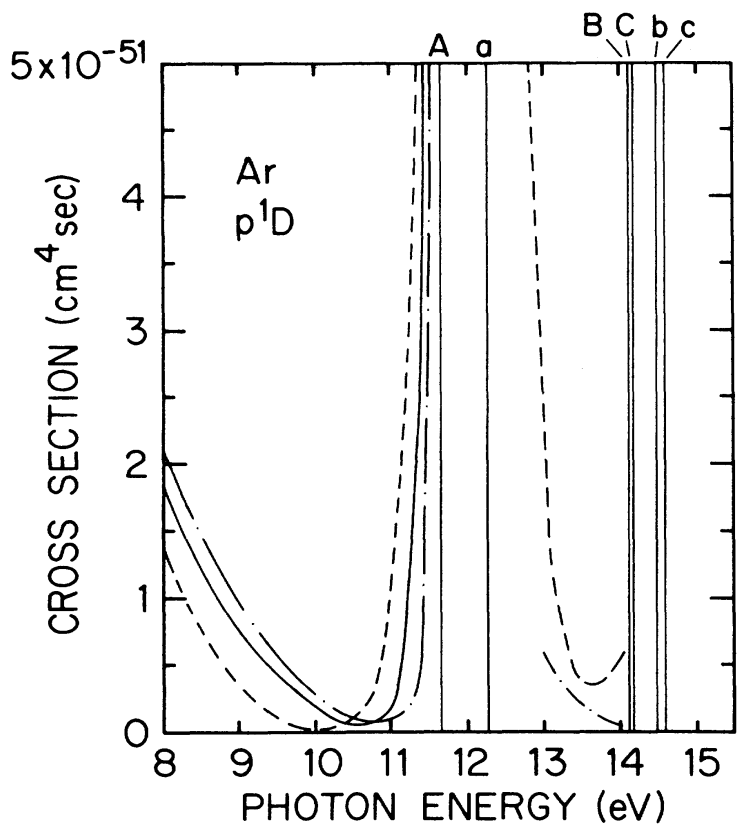

FIG. 3. Generalized two-photon cross section (length form) for the transition $\operatorname{Ar} 3 p^{6}\left({ }^{1} S\right)+2 \gamma \rightarrow \mathrm{Ar}^{+} 3 p^{5} \varepsilon p\left({ }^{1} D\right)$ for photon energies below the single-ionization threshold. Notations are the same as for Fig. 2. Note that the GSC results are not shown separately as they are nearly the same as the IIC results shown.

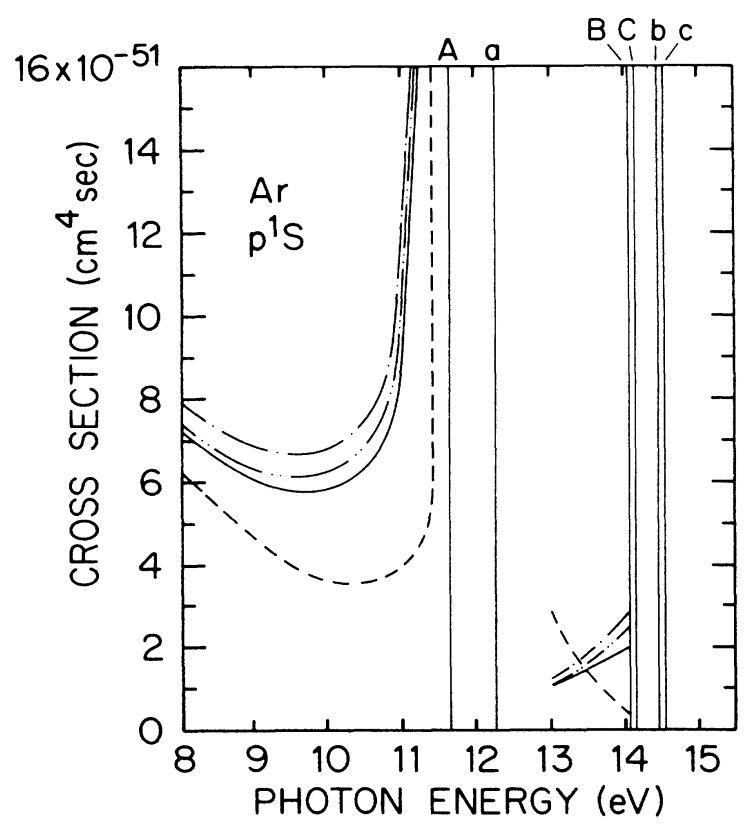

FIG. 4. Generalized two-photon cross section (length form) for the transition $\operatorname{Ar} 3 p^{6}\left({ }^{1} S\right)+2 \gamma \rightarrow \operatorname{Ar}^{+} 3 p^{5} \varepsilon p\left({ }^{1} S\right)$ for photon energies below the single-ionization threshold. Notations are the same as for Fig. 2.
(HF) approximation, we calculate the transition matrix $T(\omega)$ by including only the first term on the right in Eq. (10); the function $\psi_{l_{\lambda}}^{\lambda}$ is computed using only the first source term on the right of Eq. (13) and ignoring interchannel coupling by requiring $l^{\prime}=l$ in the sum over $l^{\prime}$ in Eq. (13). At the next level of approximation, we include ground-state correlations (GSC) by calculating the transition matrix $T(\omega)$ using both terms on the right in Eq. (10); the functions $\psi_{l_{\lambda}}^{\lambda}$ and $\bar{\phi}_{d}^{\lambda}$ are obtained as the solutions of Eqs. (13) and (14), respectively, but in Eq. (13) interchannel coupling is ignored by requiring $l^{\prime}=l$ in the sum over $l^{\prime}$. The third level of approximation includes both ground-state correlations and intermediate-state interchannel coupling (IIC); the transition matrix $T(\omega)$ is computed from Eqs. (10), (13), and (14) as they are written. Lastly, in the fourth level of approximation, we add the effect of the electron scattering interactions (ESI), which are discussed in Sec. IV, by calculating the transition matrix $T(\omega)$ from Eqs. (19), (13), and (14).

\section{Discussion of effects of electron correlations}

Examination of Figs. 2-4 shows that in the region between threshold and the $\operatorname{Ar} 3 p^{6} 4 s\left({ }^{1} P\right)$ intermediate-state resonance, all of the approximations which improve upon the HF approximation lower the generalized cross section. Ground-state correlations and intermediate-state interchannel coupling correlations reduce the generalized cross section by about $15 \%$. The electron-scattering interactions reduce the cross sections significantly more (e.g., by approximately a factor of 2 in some cases at certain photon energies). This large near-threshold reduction in the cross section due to the electron-scattering interactions is in agreement with similar findings of L'Huillier and Wen$\operatorname{din}^{17}$ for two-photon ionization of the $5 p$-subshell of xenon.

Our calculations allow some additional general conclusions, which are not illustrated in Figs. 2-4, on the importance of different kinds of ground-state correlations. We have found that inclusion of $l=3$ doubly excited configurations in the initial state [cf. Eq. (9a)], whose effect enters through the second source term on the right in Eq. (14), is unnecessary. Similarly, we have found that inclusion of doubly excited configurations in the intermediate state [cf. Eq. (9b)], whose effect enters through the function $\bar{\phi}_{d}^{\lambda}$ [cf. Eq. (12)], is negligible. Both effects, which influence the cross section through the second term in the transition matrix $T(\omega)$ in Eq. (10), change the cross sections on the order of $2 \%$. This finding implies that virtual pairs of excited electrons are important only to describe the interaction of photons with the atom, as in the case of single-photon ionization processes. The effect of these virtually excited electrons' being further excited by the incident photons (as opposed to being de-excited) is negligible. Thus theoretical treatments of higher-order multiphoton processes can probably use HF-approximation wave functions for all photoelectron intermediate states except the first one. The function $\psi_{l_{\lambda}}^{\lambda}$ calculated from $\mathrm{Eq}$. (13), is required for the first intermediate state and for all correction terms involving photons interacting with the atomic or ionic core (as for, e.g., the electronscattering interactions discussed in Sec. IV). 


\section{Discussion regarding intermediate-state resonances}

In each of Figs. 2-4 we have indicated the location of the Ar $3 p^{5} 4 s\left({ }^{1} P\right)$, Ar $3 p^{5} 5 s\left({ }^{1} P\right)$, and $\mathrm{Ar} 3 p^{5} 3 d\left({ }^{1} P\right)$ intermediate-state resonances by vertical lines. For each resonance we have indicated both the experimentally observed photon energy ${ }^{48}$ as well as our predicted HF energy. The energy values are given in Table III. In our calculations, HF energies were used whenever energies were explicitly required. However, in order to facilitate comparison with both experiment and with Ref. 13, we have used the experimental $3 p$ ionization threshold, ${ }^{49} 0.579$ a.u. $(15.76 \mathrm{eV})$, rather than the HF one, 0.591 a.u. $(16.08 \mathrm{eV})$, to compute the generalized cross section [Eq. (23)]. Thus at any given $\omega$ in our figures, the kinetic energy we have used for our final-state wave functions is $k^{2} / 2=2 \omega$ -0.579 a.u.

With one exception, we have not given predictions for the generalized two-photon cross sections in the vicinity of the intermediate state $4 s\left({ }^{1} \mathrm{P}\right), 5 s\left({ }^{1} \mathrm{P}\right)$, and $3 d\left({ }^{1} \mathrm{P}\right)$ resonances. The reason is that the Dalgarno-Lewis ${ }^{27}$ procedure we use to solve Eqs. (13) and (14) does not converge at energies close to these resonances. Chang and Poe $^{50}$ have shown how to handle this difficulty when one knows the energies of these resonances, as in the purely HF calculations they carry out. In our case, however, the correlations we have included are expected to improve the HF resonance energies to be closer to the experimental energies. However, we have chosen not to focus on the determination of these resonance energies, which is really a rather different problem. We therefore simply indicate both the experimental and the HF resonance energies in our figures and have not tried to give predictions for the cross sections in these regions. The one exception is the $f\left({ }^{1} D\right)$ channel cross section, for which we have predicted approximately the cross section in the region of the intermediate-state resonances (cf. Fig. 5) in order to compare with the work of Ref. 13, as discussed in Sec. V C.

Finally, we mention here one particularly striking effect of intermediate-state interchannel interactions on the theoretically predicted resonance structure of the $f\left({ }^{1} D\right)$ channel cross section, shown in Figs. 2 and 5. After absorption of the first photon by the $3 p$ subshell, there are two intermediate-state $\left({ }^{1} P\right)$ channels:

$$
\begin{aligned}
\operatorname{Ar} 3 p 6\left({ }^{1} S\right)+\gamma & \rightarrow \operatorname{Ar}^{+} 3 p^{5}\left({ }^{2} P\right) \varepsilon d\left({ }^{1} P\right) \\
& \rightarrow \mathrm{Ar}^{+} 3 p^{5}\left({ }^{2} P\right) \varepsilon s\left({ }^{1} P\right)
\end{aligned}
$$

Now without interchannel interactions between the $d\left({ }^{1} P\right)$

TABLE III. Energies of the lowest three intermediate-state resonances.

\begin{tabular}{lcc}
\hline \hline $\begin{array}{c}\text { Resonance } \\
\text { configuration }\end{array}$ & \multicolumn{2}{c}{ Energy above Ar ground state $(\mathrm{eV})$} \\
\hline $\operatorname{Ar} 3 p^{5} 4 s\left({ }^{1} P\right)$ & 11.62 & $\mathrm{HF}$ \\
Er $3 p^{5} 5 s\left({ }^{1} P\right)$ & 14.09 & 12.23 \\
$\operatorname{Ar~} 3 p^{5} 3 d\left({ }^{1} P\right)$ & 14.15 & 14.47 \\
\hline \hline
\end{tabular}

${ }^{\mathrm{a}}$ See Ref. 48.

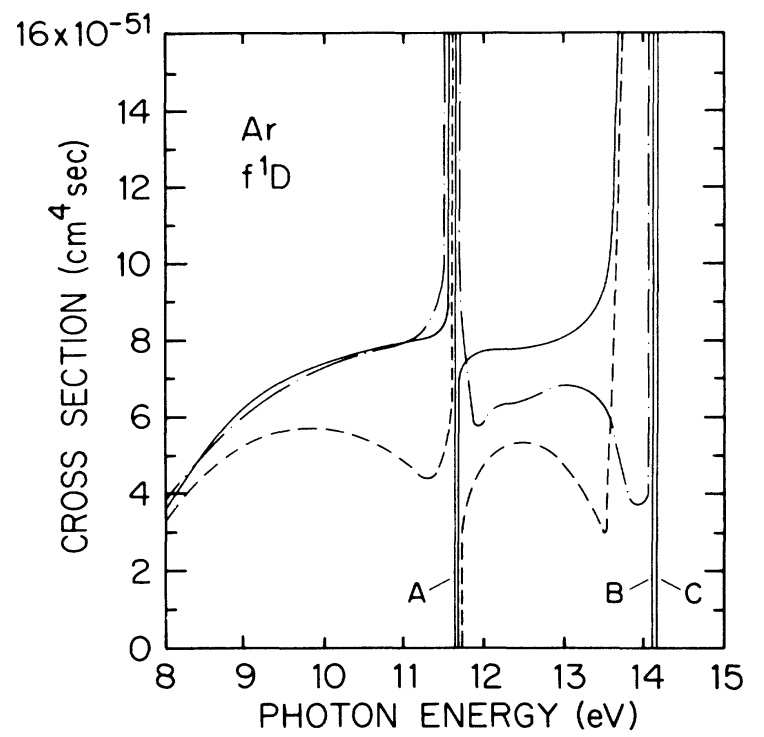

FIG. 5. Generalized two-photon cross section (length form) for the transition $\operatorname{Ar} 3 p^{6}\left({ }^{1} S\right)+2 \gamma \rightarrow \mathrm{Ar}^{+} 3 p^{5} \varepsilon f\left({ }^{1} D\right)$ for photon energies below the single-ionization threshold. Our third (IIC) and fourth (ESI) level approximation results, described in the caption to Fig. 2, have been shifted to give resonance profiles at the observed experimental energies, denoted by $A, B$, and $C$, as in Fig. 2. Solid curve, shifted IIC results; dashed curve, shifted ESI results; dot-dash curve, "LGI" results of Pindzola and Kelly (Ref. 13).

and $s\left({ }^{1} P\right)$ channels in Eq. $(25)$, the $s\left({ }^{1} P\right)$ channel does not contribute to the $f\left({ }^{1} D\right)$ final state in Eq. (20a). Hence, as shown in Fig. 2, at the HF level as well as in the case where only ground-state correlations are introduced, the corresponding theoretical two-photon cross section passes smoothly through the region of the $4 s\left({ }^{1} P\right)$ resonance. Only when such interchannel interactions are introduced does one see an effect on the calculated two-photon cross sections, as shown in Figs. 2 and 5. (This effect has been pointed out also by Pindzola and Kelly. ${ }^{13}$ )

\section{Comparisons with other calculations}

There have been two detailed calculations of the twophoton cross section of the $3 p$ subshell of argon which treat many of the electron correlation effects considered here using different theoretical approaches. These are the many-body perturbation theory (MBPT) calculation of Pindzola and Kelly ${ }^{13}$ and the approximate random-phase approximation (RPA) calculation of Moccia, Rahman, and Rizzo. ${ }^{15}$ We compare our results with each of these works as well as with the recent central potential model calculations of McGuire. ${ }^{34}$

\section{Generalized two-photon cross section for the $f\left({ }^{1} D\right)$ channel}

Pindzola and Kelly ${ }^{13}$ have predicted the cross sections for each of the individual channels in Eq. (20). However, they treat electron correlations only for the $f\left({ }^{1} D\right)$ channel. 
For this reason, we compare our results with theirs in detail only for this channel. In order to make a fair comparison of our results with theirs, we have had to take into account that in Ref. 13 the intermediate-state summation [which we handle by the Dalgarno-Lewis differential equation technique ${ }^{27}$ (cf. Sec. II A above)] is carried out directly using the experimental resonance energies given in Table III. Therefore, we have shifted approximately the energy position of our calculated resonance profiles for this channel to the experimental resonance energies in Table II using the techniques described by Chang and Poe. ${ }^{50}$ The results for our third and fourth levels of approximation for the treatment of electron correlations (cf. Sec. V B 1 above) are compared in Fig. 5 with the "LGI" results of Ref. 13. ${ }^{51}$

Our third level of approximation (IIC), which treats ground-state correlations and intermediate-state interchannel interactions, is theoretically comparable to the "LGI" results of Pindzola and Kelly. ${ }^{13}$ That is, both calculations include the same major types of electron correlation effects. One sees that the predictions by these two very different methods are essentially identical in the region below the $4 s\left({ }^{1} P\right)$ intermediate-state resonance. Between the $4 s\left({ }^{1} P\right)$ and $5 s\left({ }^{1} P\right)$ resonance energies, these two theoretical predictions differ. One possible explanation may be the much greater difficulty we had for the $5 s\left({ }^{1} P\right)$ and $3 d\left({ }^{1} P\right)$ intermediate-state resonances in shifting our curves approximately to the experimental resonance energies that are used in the calculations of Ref. 13.

The electron scattering interactions (ESI), which we treat in our fourth level of approximation, and which Ref. 13 does not treat, reduces our predicted cross section by an increasing amount as one approaches the $4 s\left({ }^{1} P\right)$ resonance from below. It reduces the cross section as well between the $4 s\left({ }^{1} P\right)$ and $5 s\left({ }^{1} P\right)$ resonances. As mentioned already, these ESI predictions for argon are consistent with those in Ref. 17 for the $5 p$ subshell of xenon.

\section{Total two-photon cross section for circularly polarized light}

McGuire ${ }^{34}$ and Moccia et al. ${ }^{15}$ have not published their predictions for the individual channels in Eq. (20), but rather they give the total two-photon cross sections that experimentalists are most likely to measure. We examine here the predictions for the total two-photon cross section for circularly polarized photons. From Table II we see that the $p\left({ }^{1} S\right)$ channel in Eq. (20c) does not contribute and that the $p\left({ }^{1} D\right)$ and $f\left({ }^{1} D\right)$ cross sections, shown in Figs. 2 and 3 for linearly polarized light, need only be multiplied by $\frac{3}{2}$ to obtain the corresponding cross sections for circularly polarized light.

Our IIC and ESI length form results (cf. Sec. V B 1 above) are shown together with the length form results of McGuire, ${ }^{34}$ Moccia et al., ${ }^{15}$ and Pindzola and Kelly ${ }^{13,51}$ in Fig. 6. The results for Pindzola and Kelly ${ }^{13}$ were obtained by multiplying by 1.5 the sum of their HF results for the $p\left({ }^{1} D\right)$ channel and their "LGI" results for the $f\left({ }^{1} D\right)$ channel. Our IIC results are nearly identical to the resulting predictions of Pindzola and Kelly. ${ }^{13}$ [Note that although Pindzola and Kelly ${ }^{13}$ have not treated electron

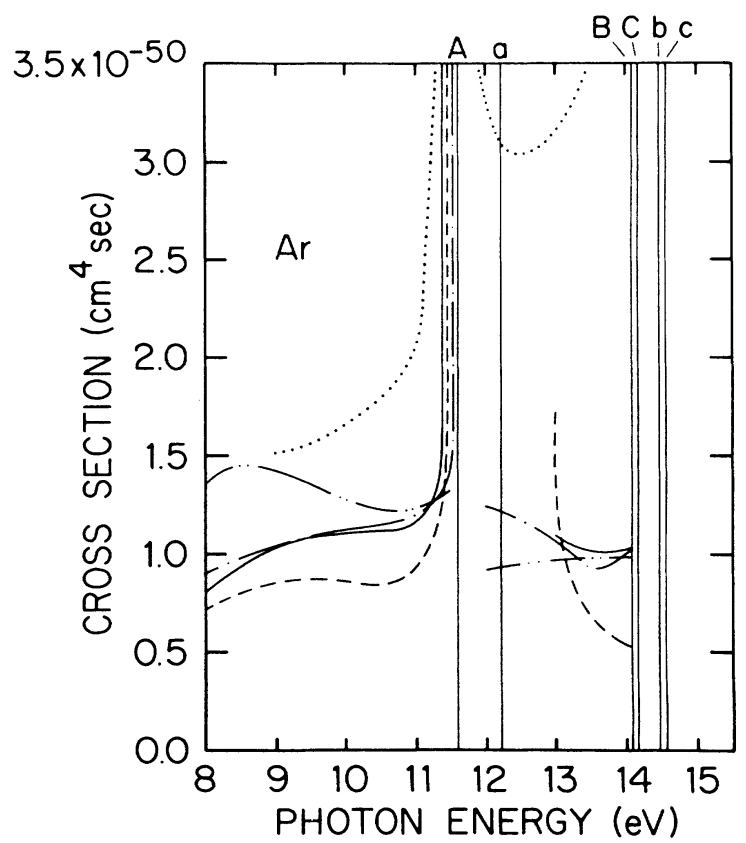

FIG. 6. Generalized two-photon total cross section (length form) for circularly polarized photons. Solid curve, present IIC results; dashed curve, present ESI results; dotted curve, central potential model results of McGuire (Ref. 34); dot-dash curve, MBPT results of Pindzola and Kelly (Ref. 13); dash-double-dot curve, approximate RPA results of Moccia, Rahman, and Rizzo (Ref. 15). See text for descriptions of our IIC and ESI calculations (in Sec. V B 1) and for descriptions of the results of other authors (in Sec. V C 2).

correlations for the $p\left({ }^{1} D\right)$ channel, as shown by our results in Fig. 3, the correlation effects for this channel are much less significant than for the $f\left({ }^{1} D\right)$ channel, which is in any case much larger.] The central potential model predictions of McGuire ${ }^{34}$ appear to be close to the approximate RPA predictions of Moccia et al. ${ }^{15}$ near $9 \mathrm{eV}$, but for higher photon energies they are much higher than the results of all of the other calculations. The predictions of Moccia et al. ${ }^{15}$ shown in Fig. 6 were obtained from their total cross section for linearly polarized light and their circular to linear cross-section branching ratio. Their results are considerably above our IIC results and the results of Pindzola and Kelly ${ }^{13}$ from threshold to the $4 s\left({ }^{1} P\right)$ resonance and they drop below these other results above the $4 s\left({ }^{1} P\right)$ resonance. Possible explanations for these differences are, firstly, their use of relaxed core wave functions as compared to our use of frozen core wave functions, and secondly, their use of continuum wave functions calculated in a static exchange potential as opposed to our use of continuum HF wave functions calculated in an appropriate $V^{N-1}(L S)$ potential (cf. Sec. V A above).

\section{Total two-photon cross section for linearly polarized light}

Theoretical predictions for the total two-photon cross section for linearly polarized light are shown in Fig. 7. Our predictions in our IIC and ESI approximations (cf. Sec. V B 1) are simply the sum of the corresponding indi- 


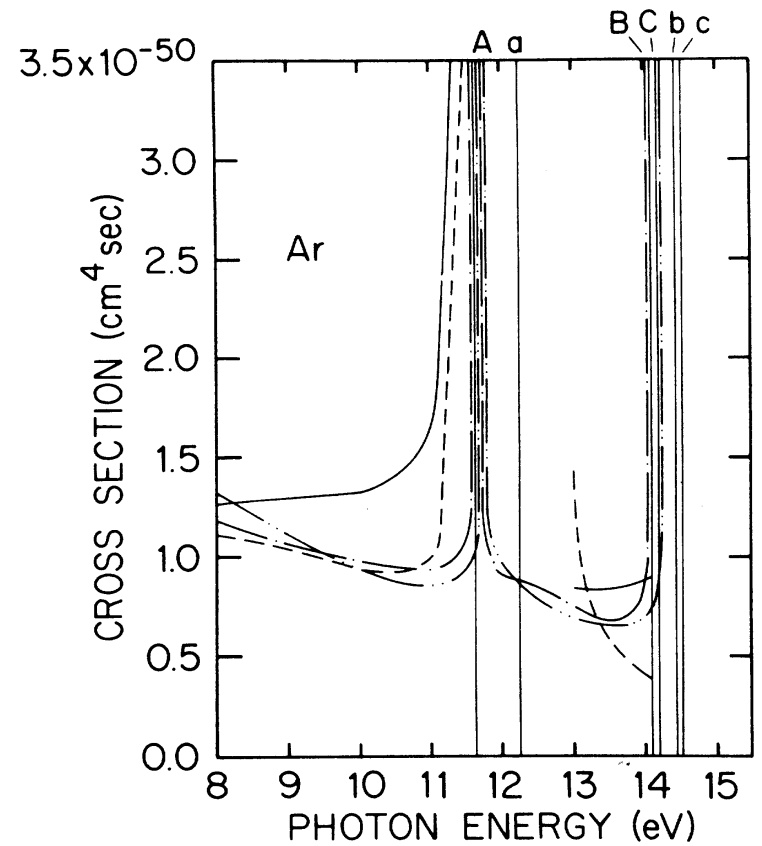

FIG. 7. Generalized two-photon total cross section (length form) for linearly polarized photons. Notation is the same as in Fig. 6.

vidual channel cross sections shown in Figs. 2-4. As before, our ESI results are significantly below our IIC results due to the strong effect of electron-scattering interactions. The results of Pindzola and Kelly ${ }^{13}$ shown are obtained as the sum of their HF predictions for the $p\left({ }^{1} D\right)$ and $p\left({ }^{1} S\right)$ channels and their "LGI" predictions for the $f\left({ }^{1} D\right)$ channels. Generally, we would expect their results as obtained in this way to be close to our IIC results, as in the case of circularly polarized light. The fact that their results are significantly lower than our IIC results (and in fact are very close to our ESI results) has been traced to differences, which are not understood, in our respective predictions for the $p\left({ }^{1} S\right)$ channel cross sections at the HF level. ${ }^{52}$

Finally, the results of Moccia, Rahman, and Rizzo ${ }^{15}$ appear to agree with both our ESI results and the results of Pindzola and Kelly. ${ }^{13}$ However, when one notes that the total cross section for circular polarization of Moccia et al. ${ }^{15}$ is larger than either one of ours or the one of Pindzola and Kelly (cf. Fig. 6), one must conclude that the $p\left({ }^{1} S\right)$ cross section of Moccia et al. ${ }^{15}$ must be smaller than either one of ours or the one of Pindzola and Kelly in order to have the total cross sections for linear polarization agree.

We conclude, therefore, that all three calculations differ significantly in their predictions for the $p\left({ }^{1} S\right)$ channel cross section. These differences are hidden by the apparently fortuitous agreement of three of the cross sections in Fig. 7 for the total two-photon cross section for linear polarization.

\section{CONCLUSIONS}

Our application to the $3 p$ subshell of argon of a transition-matrix-theory approach for two-photon ioniza- tion processes allows two main conclusions regarding electron correlation effects. The first is that the major correlation effects of virtually excited electron pairs are the same as those needed to describe the photon-atom interaction in ordinary one-photon ionization processes. In particular, we find that whenever a photon interacts with the atom or ion, virtually excited electron pairs must be included, since their de-excitation in the photoabsorption process has a significant effect on the transition amplitude. The further excitation of these pairs to intermediate states, however, has negligible effects. This finding greatly simplifies the extension of our theory to higher-order multiphoton processes.

The second conclusion is that the electron-scattering interactions, described in Sec. IV, reduce the two-photon cross section substantially below the single-photon ionization threshold, in agreement with similar findings for xenon of L'Huillier and Wendin. ${ }^{17}$ In other words, there is strong competition between two mechanisms of ionization: The first is the one in which each of the two photons acts on the same photoelectron; the second is the one in which each of the photons excites a different photoelectron and these two photoelectrons then scatter from one another in such a way that one is de-excited back to its initial state and the other goes off as the single, observable photoelectron. It is this latter mechanism, whose importance was discovered in Ref. 17 for xenon and confirmed here for argon, which is new.

With regard to the comparison of our results with those of other authors, we find the following: Firstly, our results which include the electron-scattering interactions give generally lower cross sections than those of others. Secondly, our results without these electron-scattering interactions (but including ground-state correlations and intermediate-state interchannel coupling) are in excellent agreement with the MBPT calculation of Pindzola and Kelly ${ }^{13}$ for the $f\left({ }^{1} D\right)$ channel and the total two-photon cross section for circularly polarized photons. Thirdly, none of the calculations appear to agree on the $p\left({ }^{1} S\right)$ final-state channel cross section, although this fact is obscured by fortuitous agreement of three of the theoretical total two-photon cross sections for linearly polarized light. Experimentalists should be aware that the theoretical predictions are probably more reliable for the total twophoton cross section for circularly polarized light, since the $p\left({ }^{1} S\right)$ final state is not populated by circularly polarized photons. Fourthly, central potential model calculations give a much larger total two-photon cross section for the $3 p$ subshell of argon than do calculations which either start with a $\mathrm{HF}$ representation or else treat some portion of electron correlation effects.

\section{ACKNOWLEDGMENTS}

We wish to express our gratitude to Dr. Peter Zoller for extensive discussions on the theoretical formulation, to Professor Charlotte Froese Fischer for assistance in using her multiconfiguration Hartree-Fock code (Ref. 37), to Professor Michael Pindzola for discussions concerning his many-body-perturbation-theory calculation for two- 
photon ionization of argon (Ref. 13), and to Dr. Anne L'Huillier and Dr. Göran Wendin for discussions concerning their random-phase-approximation calculation for two-photon ionization of xenon (Ref. 17). This work was supported in part by Osterreichische Forschungsgemeinschaft Grant No. 06/0088 and by U.S. National Science Foundation Grant Nos. PHY-8305739 and PHY8601429.
*Permanent address: Department of Physics and Astronomy, The University of Nebraska, Lincoln, NE 68588-0111.

†Present address: Institute of Atomic and Molecular Sciences, Academia Sinica, P.O. Box 23-166, Taipei, Taiwan 107, Republic of China.

${ }^{1}$ For reviews of multiphoton ionization, see the various contributions in Multiphoton Ionization of Atoms, edited by S. L. Chin and P. Lambropoulos (Academic, New York, 1984).

${ }^{2}$ A. L'Huillier, L. A. Lompre, G. Mainfray, and C. Manus, Phys. Rev. A 27, 2503 (1983); J. Phys. B 16, 1363 (1983).

${ }^{3}$ K. Boyer, H. Egger, T. S. Luk, H. Pummer, and C. K. Rhodes, J. Opt. Soc. Am. B 1, 3 (1984).

${ }^{4}$ C. K. Rhodes, in Fundamentals of Laser Interactions, edited by F. Ehlotzky (Springer, Berlin, 1985), pp. 111-124; Science 220, 1345 (1985).

${ }^{5}$ D. Feldmann, H.-J. Krautwald, and K. H. Welge, Ref. 1, pp. 223-233.

${ }^{6}$ P. Kruit, J. Kimman, H. G. Muller, and M. J. van der Wiel, Phys. Rev. A 28, 248 (1983).

${ }^{7}$ A. Agostini and G. Petite, in Multiphoton Processes, edited by P. Lambropoulos and S. J. Smith (Springer, Berlin, 1984), pp. 13-22.

${ }^{8}$ A. F. Starace, Appl. Opt. 19, 4051 (1980).

${ }^{9}$ A. F. Starace, Theory of Atomic Photoionization, Vol. 31 of Handbuch der Physik, edited by W. Mehlhorn (SpringerVerlag, Berlin, 1982), pp. 1-121.

${ }^{10}$ A. F. Starace, in Fundamental Processes in Energetic Atomic Collisions, edited by H. O. Lutz, J. S. Briggs, and H. Kleinpoppin (Plenum, New York, 1983), pp. 69-110.

${ }^{11}$ See, e.g., M. Crance, Ref. 1, pp. 65-108.

${ }^{12}$ G. A. Victor, Proc. Phys. Soc. London 91, 825 (1967).

${ }^{13}$ M. S. Pindzola and H. P. Kelly, Phys. Rev. A 11, 1543 (1975)

${ }^{14}$ B. Ritchie, Phys. Rev. A 16, 2080 (1977).

${ }^{15}$ R. Moccia, N. K. Rahman, and A. Rizzo, J. Phys. B 16, 2737 (1983).

${ }^{16}$ A. L'Huillier, L. Jönsson, and G. Wendin, Phys. Rev. A 33, 3938 (1986).

${ }^{17}$ A. L'Huillier and G. Wendin, J. Phys. B 20, L37 (1987).

${ }^{18}$ M. G. J. Fink and P. Zoller, J. Phys. B 18, L373 (1985).

${ }^{19}$ M. Crance and M. Aymar, J. Phys. B 18, 3529 (1985).

${ }^{20}$ P. Lambropoulos, Phys. Rev. Lett. 55, 2141 (1985).

${ }^{21}$ X. D. Mu, T. Åberg, A. Blomberg, and B. Craseman, Phys. Rev. Lett. 56, 1909 (1986).

${ }^{22}$ G. Wendin, L. Jönsson, and A. L'Huillier, Phys. Rev. Lett. 56, 1241 (1986).

${ }^{23}$ A. L. Robinson, Science 232, 1193 (1986).

${ }^{24}$ M. Crance, Phys. Rep. 144, 117 (1987).

${ }^{25}$ A. F. Starace, Phys. Scr. Topical Volume T 17 (1987).

${ }^{26}$ A. W. McCown, M. N. Ediger, and J. G. Eden, Phys. Rev. A 26, 3318 (1982).

${ }^{27}$ A. Dalgarno and J. T. Lewis, Proc. R. Soc. London, Sect. A 233, 70 (1955).

${ }^{28}$ T. N. Chang and U. Fano, Phys. Rev. A 13, 263 (1976); 13, 282 (1976).
${ }^{29}$ T. N. Chang, Phys. Rev. A 15, 2392 (1977); 16, 1171 (1977); 18, 1448 (1978); 20, 291 (1979); Phys. Scr. 21, 463 (1980); J. Phys. B 13, L551 (1980).

${ }^{30}$ A. F. Starace and S. Shahabi, Phys. Rev. A 25, 2135 (1982).

${ }^{31}$ S. Shahabi, A. F. Starace, and T. N. Chang, Phys. Rev. A 30, 1819 (1984).

${ }^{32}$ S. Shahabi and A. F. Starace, Phys. Rev. A 33, 2111 (1986).

${ }^{33}$ M. Ya. Amusia and N. A. Cherepkov, Case Stud. At. Phys. 5, 47 (1975).

${ }^{34}$ E. J. McGuire, Phys. Rev. A 24, 835 (1981).

${ }^{35}$ A. F. Starace and P. Zoller, in Fundamentals of Laser Interactions, Vol. 229 of Lecture Notes in Physics, edited by F. Ehlotzky (Springer, Berlin, 1985), pp. 292-293.

${ }^{36}$ T. F. Jiang and A. F. Starace, Bull. Am. Phys. Soc. 31, 943 (1986).

${ }^{37}$ C. Froese Fischer, Comput. Phys. Commun. 4, 107 (1972); The Hartree-Fock Method for Atoms (Wiley, New York, 1977).

38J. R. Swanson and L. Armstrong, Jr., Phys. Rev. A 15, 661 (1977); 16, 1117 (1977)

${ }^{39}$ Cf. Eqs. (14)-(16) of Ref. 30.

${ }^{40}$ C. Froese Fischer, Comput. Phys. Commun. 1, 151 (1969); 4, 107 (1972).

${ }^{41}$ See Ref. 9, p. 73, and references therein.

${ }^{42}$ R. T. Pu and E. S. Chang, Phys. Rev. 151, 31 (1966).

${ }^{43}$ H. J. Silverstone and M. L. Yin, J. Chem. Phys. 49, 2026 (1968).

${ }^{44}$ S. Huzinaga and C. Arnau, Phys. Rev. A 1, 1285 (1970).

${ }^{45}$ See Ref. 31 , Sec. III B 1.

${ }^{46}$ P. de A. P. Martins, J. Phys. B 1154 (1968); Proc. Cambridge Philos. Soc. 69, 167 (1971).

${ }^{47}$ P. Lambropoulos, Adv. At. Mol. Phys. 12, 87 (1976).

${ }^{48} \mathrm{We}$ quote in Table III the experimental resonance energies used in the calculation of Pindzola and Kelly (Ref. 13) in order to facilitate the comparison with their results (shown in our Fig. 5). They use the experimental wave numbers given for the levels designated (in $j l$ coupling) by $4 s\left[1 \frac{1}{2}\right]^{\circ} J=1$, $5 s\left[1 \frac{1}{2}\right]^{\circ} J=1$, and $3 d\left[1 \frac{1}{2}\right]^{\circ} J=1$ in C. E. Moore, Atomic Energy Levels, Nat. Stand. Ref. Data Ser., Natl. Bur. Stand. (U.S.) Circ. No. 35 (U.S. GPO, Washington, D.C., 1971), Vol. I, p. 212. The wave numbers there have been converted to $\mathrm{eV}$ using the factor $1 \mathrm{eV}=8065.73 \mathrm{~cm}^{-1}$.

${ }^{49}$ C. E. Moore, Ionization Potentials and Ionization Limits Derived from the Analyses of Optical Spectra, Nat. Stand. Ref. Data Ser., Nat. Bur. Stand. (U.S.) Circ. No. 34 (U.S. GPO, Washington, D.C., 1970), p. 6 b.

${ }^{50}$ T. N. Chang and R. T. Poe, J. Phys. B 9, L311 (1976); Phys. Rev. A 16, 606 (1977).

${ }^{51}$ The generalized cross sections in Ref. 13 are given in units of $10^{-18} \mathrm{~cm}^{2}$ per unit flux (a.u.). The conversion factor to the more standard units, $\mathrm{cm}^{4} \mathrm{sec}$, is $6.7734 \times 10^{-34}$. Note also that the notation "LGI" is defined in Ref. 13 to denote "length curves [which] include contributions from diagrams leading to the $4 s^{1} P$ and $5 s^{1} P$ resonances as well as thirdorder intrashell ground-state correlations." 
${ }^{52}$ These differences in the $p\left({ }^{1} S\right)$ cross section stem, we believe, from differences in the $p\left({ }^{1} S\right)$ final-state radial wave functions, which we describe here for the record. At the HF level, the only differences in technique between our calculations and those in Ref. 13 of which we are aware concern the orthogonalization of the $p\left({ }^{1} S\right)$ wave function to the bound $2 p$ and $3 p$ wave functions. In our calculations, we carry out this orthogonalization for both the $p\left({ }^{1} D\right)$ and $p\left({ }^{1} S\right)$ orbitals using the techniques of Refs. 42-44. Pindzola and Kelly use these same techniques for the $p\left({ }^{1} D\right)$ orbitals but use Schmidt orthogonalization for the $p\left({ }^{1} S\right)$ orbitals. Another bit of evidence that our $p\left({ }^{1} S\right)$ wave functions and those of Ref. 13 differ is given by Table I of Ref. 13, which lists matrix elements of $z^{2}$ between the $3 p$ orbital and the three types of final-state orbitals, $f\left({ }^{1} D\right), p\left({ }^{1} D\right)$, and $p\left({ }^{1} S\right)$. We agree exactly on the matrix elements for $f\left({ }^{1} D\right)$ and $p\left({ }^{1} D\right)$, but our respective wave functions give different values of the matrix elements for $p\left({ }^{1} S\right)$. 